Published in Proceedings of X Workshop on Agents Applied in Health Care, A2HC 2017, São Paulo, Brazil, 8-9 May 2017, which should be cited to refer to this work.

\title{
Agent-based systems for telerehabilitation: strengths, limitations and future challenges
}

\author{
Davide Calvaresi ${ }^{1,2}$, Michael Schumacher ${ }^{2}$, Mauro Marinoni ${ }^{1}$, Roger Hilfiker ${ }^{3}$, \\ Aldo F. Dragoni ${ }^{3}$, and Giorgio Buttazzo ${ }^{1}$ \\ 1 Scuola Superiore Sant'Anna,Pisa, Italy \\ 2 University of Applied Sciences Western Switzerland, Sierre, Switzerland \\ 3 Universitá Politecnica delle Marche, Ancona, Italy \\ d.calvaresi,m.marinoni,g.buttazzo\{@sssup.it\}, a.f.dragoni@univpm.it, \\ michael.schumacher@hevs.ch
}

\begin{abstract}
Telerehabilitation in older adults is most needed in the patient environments, rather than in formal ambulatories or hospitals. Supporting such practices brings significant advantages to patients, their family, formal and informal caregivers, clinicians, and researchers. Several techniques and technologies have been developed aiming at facilitating and enhancing the effectiveness of telerehabilitation. This paper gives a quick overview of the state of the art, investigating video-based, wearable, robotic, distributed, and gamified telerehabilitation solutions. In particular, agent-based solutions are analyzed and discussed addressing strength, limitations, and future challenges. Elaborating on functional requirements expressed by professional physiotherapists and researchers, the need for extending multi-agent systems (MAS) peculiarities at the sensing level in wearable solutions establishes new research challenges. Employed in cyber-physical scenarios with users-sensors and sensorssensors interactions, MAS are requested to handle timing constraints, scarcity of resources and new communication means, which are crucial for providing real-time feedback and coaching.
\end{abstract}

Keywords: Multi-agent systems, wearable Multi-agent systems, Realtime Multi-agent systems, telerehabilitation, real-time systems, review, MAS.

\section{Introduction}

Healthcare institutions are facing the strain of a significantly larger elderly population [1]. Lengthening life expectancy is met by an increasing demand for medical and technological contributions to extend the "good-health", and disabilityfree period.

The major factor catalyzing the elderly's impairing process is the progressive reduction of mobility, due to the natural aging process, inactivity, diseases such as osteoarthritis, stroke or other neurological conditions, falls with its consequences, such as fear of falls (leading to inactivity), or fractures (needing surgery).Despite the emergence of less-invasive surgical techniques, postintervention rehabilitation still requires extended periods and tailored therapies, 
which usually involve complications. Performing traditional rehabilitative practices is leading to a significant increase in public-health costs and, in some cases, a lack of resources, thus worsening the services' quality. Rehabilitation is often a long process and needs to be sustained long after the end of the acute care. Simplifying the access to health services [2] can raise the number of patients, maintaining (or even increasing) the quality of care. For example, patients requiring support, such as continuous or selective monitoring, can benefit from systems that automatically transmit the information gathered in their domestic environment to the health clinics, thus enabling telemonitoring on their health conditions [3].

Although in traditional solutions telemonitoring is a self-contained practice limited to passively observing the patients, the need for remote sensing is crucially coupled with the need for coaching older adults in their daily living $[4$, $5]$.

For example, a critical activity such as telerehabilitation cannot be limited to observing the patients' behaviors. Indeed, patient adherence and acceptability of rehabilitative practices need to be actively enhanced, overcoming pitfalls due to motor (e.g., endurance), non-motor (e.g., fatigue, pain, dysautonomic symptoms, and motivational), and cognitive deficits. According to Rodriguez et al. [6], telerehabilitation can be formally defined as:

"the application of telecommunication, remote sensing and operation technologies, and computing technologies to assist with the provision of medical rehabilitation services at a distance."

Patients, physiotherapists, and health institutes can gain several benefits from an extensive adoption of telerehabilitation systems [7]. Considering the economical point of view, Mozaffarian et al. [8] figured out that the total cost of stroke in the US was estimable to be 34.3 billion dollars in 2008 , rising up to 69.1 billion dollars in 2016.

Even though to date they are not precisely quantifiable due to insufficient evidence [9], Mutingi et al. [10] presented as "inevitable advantages" (i) a substantial cost saving primarily due to the reduction of specialized human resources, (ii) an enhancement of patient comfort and lifestyle, and (iii) improvements of therapy and decision making processes. Moreover, Morreale et al. [11] mentioned one of the most appreciated benefits: the increase of adherence to rehabilitation protocols.

The multitude of scientific contributions fostering telerehabilitation exploits new technologies and various architectures to better understand and serve user requirements. However, due to technological or technical limitations, physiotherapists' needs have not yet been completely satisfied. To fill this gap, a system evolution is required. For example, telerehabilitation systems cannot offer the same behavior to users with diverse conditions. Viceversa, according to the environment condition, they must rather be able to adapt themselves to the user needs $[6]$.

Telerehabilitation is characterized by a very delicate equilibrium between environment, devices, and users. Thus, the capabilities such as self-adaptation, 
flexibility, and ubiquity are crucial to facilitate and promote the usability and then the actual practices.

\section{Contributions}

This paper provides the following contributions:

- It summarizes the most relevant results provided by telemonitoring solutions, with particular emphasis on multi-agent systems (MAS).

- It details the requirements expressed by the physiotherapists about rehabilitation practices, which to date require the most technological supports.

- It connects the above-mentioned requirements with the offered and potential peculiarities of the envisioned real-time multi-agent systems.

- It discusses innovative challenges for MAS, such as deploying intelligent agents in wearable sensor nodes while facing compliance to strict timing constraints.

\section{Paper structure}

The rest of the paper is organized as follows. Section 2 elaborates the state of the art providing a complete overview of both conventional and agent-based telerehabilitation systems. Section 3 presents practices and still unmet requirements expressed by professional physiotherapists. Section 4 discusses strengths and limitations of current agent-based telerehabilitation systems, introducing and detailing the future challenges to be faced by MAS to enhance performance and applicability in rehabilitation scenarios. Finally, Section 5 states our conclusions summarizing the lesson learnt and presenting some future work.

\section{State of the art}

Telerehabilitation solutions primarily target the elderly and patients from rural areas unable to reach medical centers [12]. Moreover, even in countries with excellent and capillary healthcare systems, telerehabilitation systems are firmly required. For example, in Switzerland, after a surgical intervention, only a limited number of assisted therapy sessions are provided. Thus, employing telerehabilitation systems during unassisted sessions, the follow-up can be fastened enhancing the healing process.

Telerehabilitation is often described as having three primary components (i) training and counselling, (ii) assessment and monitoring, and (iii) point of delivery. Occupational therapy, physical therapy, and speech-language therapy [11] are the most provided. However, due to the lack of adequate studies, the interpretation of particular patient groups is restricted [13].

A recovery period, usually about six to eight weeks, can follow an acute trauma (e.g., fall of a fragile elder) or surgical intervention (e.g., joint replacement). This is the most critical period for patients who are luckily still not chronic. Nevertheless, even for the latter, the scientific community provided tailored solutions to relieve pain and maintain or slowly recover physical and/or 
mental capabilities. Indeed, telerehabilitation targets not only physically impaired [14], but also cognitive impaired patients $[15,16]$ within an average age of $76(56 \sim 91)$ [13].

The broad range of available technologies enabled the development of various techniques and approaches.

The main category of applications they have generated are based on:

- video analysis - mostly involving stereoscopic cameras and image processing algorithms;

- wearable technology - mostly focusing on embedded devices and inertial sensors supported by kinematic algorithms;

- robotics - mostly focused on in monitoring and motivation involving humanoids or basic robots;

- distributed sensing - mostly involving monitoring and reasoning exploiting environmental sensors;

- gamification - mostly involving coaching techniques and persuasive technologies.

Despite the considerable availability of extremely precise and complex solutions, telerehabilitation systems have to face user (patients and physiotherapists) acceptability.

The amount of similar proposed solutions suggests that the requirements set from physiotherapists and patients have not yet been entirely met. Factors such as setup, costs, maintenance, safety, easy usage, minimal set of options and functionalities, and effectiveness primarily impact on general acceptance or refusal [13].

Iarlori et al. [17] proposed a computer-vision based system applied to patients affected by Alzheimer's disease. The diagnosis of the illness's stage is performed monitoring elderly in their private environment and analyzing personal daily-care activities. Observing the actions listed in the Direct Assessment of Functional Status (DAFS) index and detecting performance's anomalies helps to define the dementia stage. The authors analyzed teeth brushing and hair grooming exploiting a Microsoft Kinect to collect data about the actions observed, tracking and supervising the user's gestures. Thus, the patient can receive an immediate support when incorrect or incoherent behaviors are detected.

Among all of the above, to obtain clinically relevant information, wearable technology gained great relevance, being considered as the possible leader of further improvements in both preventative and rehabilitation approaches, while camera-based applications still generate concerns. A study targeting patients in an elderly-care facility revealed that the $93 \%$ of the patients accepted bodyworn sensor systems, defining them as non-invasive and not affecting normal daily activities [18].

Bergmann et al. [13] reported a surprisingly high consideration among the patients about the aesthetic of wearable sensors, mainly concerned about not appearing "stigmatized". Regarding the physiotherapists, major concerns arose for a restricted recording time due to limited storage capacity, wearability, and reliable real-time feedback. 
According to Smith et al. [19], the current wearable devices successfully employed in telerehabilitation can be classified into 3 categories:

- Microsensors - capturing health information by using small, intelligent, and low-energy active devices;

- Wrist devices - monitoring health information by using combined sensors, display, and wireless transmission in a single solution, which is very convenient for common physical activities;

- Smart clothes - capturing information by using thin and flexible health sensors, which have to be compatible with textiles, or made using textile technologies, such as new fibers with specific (mechanical, electrical and optical) properties.

For example, Cesarini et al. [20] provided a highly customizable solution for supporting therapists and patients from the pre-surgical to the rehabilitation phase. Furthermore they presented a particular implementation of a framework, involving two wearable inertial sensors and a tablet, which can precisely monitor the angular position and velocity of the knee joint. Physicians and therapists can define specific exercises and related requirements (e.g., the number of repetitions, the number of steps and angular extension) characterizing the therapy. The system guides the patient during the exercises execution providing a real-time visual feedback on the tablet and evaluating the obtained performance at the end of the session.

Another study presents a real-time feedback system of aquatic-space actions (e.g., performed by swimmers or rehabilitating patients) in the form of a functional sound, exploiting the so-called sonification procedure [21]. In particular, such a system is composed of pressure sensors placed on the palmar and dorsal sides of the swimmers hands, and a water-proof embedded system placed on the back of the swimmer. The pressure signals produced by the swimmer motion are processed by the embedded system and provided in real-time to both swimmer and trainer/therapist. Furthermore, such a system can also be exploited in the context of rehabilitation activities and has already been presented in a specialized conference on aquatic therapy [22]. Therapists have widely accepted it as a promising tool for training and recovery of motor and coordination functions.

Similar solutions, but involving robotic devices in the automation of rehabilitation procedure have been considered helpful in reducing training and rehabilitative sessions of both upper and lower extremities (well-known limitation of conventional methods) [23]. Indeed, task-oriented repetitive movements have a direct positive effect on improving muscle strength and movement in patients with neurological injuries, and automated robotics solutions can acquire a higher number of exercises' repetition compared with conventional approaches [24]. Eriksson et al. [25] realized an autonomous assistive mobile robot that provided monitoring, encouragement, and reminders to aids rehabilitating stroke patients. Navigating autonomously, it monitors the patient's activity of the extremity supposed to be rehabilitating, reminding the patient to follow the program in the case of miss-behaviors. They shown experiments involving post-stroke patients. 
The proposed approach achieved positive responses about the increasingly active and animated robot behavior. The control system they have used is behaviorbased. Such behaviors were characterized as pre- and post-conditions to provide proper real-time feedback.

Jacobs et al. [26] implemented a serious game to support arm-hand rehabilitation for stroke survivors. The main objective was to make the training effective and enjoyable. Exploiting task-oriented training principles, this game requires to manipulate every-day objects, dynamically adapting its difficulty based on the patient's performance. Both physical and cognitive capabilities were involved, and the authors evaluated of two stroke patients over a week.

From a technical and technological point of view, telerehabilitation systems are complex solutions, which have to face context-rich scenarios and uncertainty, handle distributed sources of information, operate in highly dynamic environments with mutual interdependencies and sophisticated distributed controls.

Although classic approaches have been shown as potentially effective, they lack in crucial features such as compatibility, collaboration, coordination, and communication [6].

Indeed, Miranda et al. [27] refer to common incompatibility problems such as data formats (e.g., storing format of 3D images) and different communication protocols. Such systems are either subject at an inevitable abandon, or require integrative upgrades (often unfeasible or requiring a worthless/unaffordable effort).

Therefore, studies such as Bergenti et al. [28] consider multi-agent systems (MAS) a suitable "technology" to realize such applications. Section 2.1 presents the most relevant agent-based telerehabilitation systems.

\subsection{MAS for telerehabilitation}

MAS are composed of several agents which are able to communicate with their neighborhoods for computational and decision-making tasks. The agents can share their information using the network interfaces to concur reaching a shared goal. The goal might be consensus, synchronization, or surveillance [23]. Due to these characteristics, MAS have been adopted in several rehabilitation solutions which tried to cope with physical and cognitive rehabilitation or providing specialized models or tools.

\section{Physical rehabilitation}

Roda et al. [29] treated elderly motor impairment employing specific devices to control patients movements. Exploiting techniques typical of the Ambient Intelligence $(\mathrm{AmI})$, they proposed a context-aware system integrating diverse devices. Thus, the MAS can react accordingly to the context, supporting physiotherapists in developing new, or adapting already existing therapies precisely tailored to the actual patient needs. Using a Microsoft Kinect, all the motor tasks performed by a patient during the rehabilitation are under control. Moreover, employing third-party sensors they were able to gather oxygen level, posture, gesture, stress, BPM, and mood. Combining such values indexes such as pain or 
fatigue could also be detected. Physiotherapists expressed fuzzy rules, for example providing a natural way to express how transitions should be made by using linguistic values rather than numerical values. A specific agent equipped with an inference engine elaborates such data while respecting isolation and privacy requirements.

Performing cardiac rehabilitation during its second (sub-acute) and third (intensive outpatient therapy) phase, a large amount of cardiac data (complex and arguably) has to be analyzed in a short period of time. The system proposed by Mesa et al. [30] provides support in data analysis, event classification, and visualization. Such a MAS has been involved in rehabilitative tests such as (i) walking on a treadmill at different speeds and with different slopes; (ii) cycling on a stationary bike at different speeds; (iii) upper body workout; and (iv) lower body workout.

Data and context awareness is considered paramount to establish actual collaboration while interacting with remote participants. Dealing with rehabilitation systems magnifies this challenge. Hence, for both cognitive and physical rehabilitating users, the information awareness is a crucial element to provide patients with a rehabilitation path as tailored as possible. [31]

In the context of Upper Limb Rehabilitation (ULR), Rodriguez et al. [6] proposed an agent-based system to customize exercises to assist different patients providing a bespoke ULR. A noteable peculiarity of such a system is the contextawareness, which enables run-time adaptability. Hence, the system "performs" three abstract concurrent tasks: (i) while the patient is executing the exercise for the upper limb, the movements are recorded and monitored; (ii) analyzing specific patient's parameters (e.g., BPM, skin conductance) an agent is in charge of defining the level of stress/fatigue; (iii) the agent behaving as a "virtual therapist" adapts ULR's parameters such as number of repetitions and target area limits according to the current level of stress.

Felisberto et al. [32] developed a MAS that recognizes human movements, identifies human postures, and detects harmful activities in order to prevent risk situations (e.g., sudden diseases and falls). The authors exploited wireless sensor nodes and energy harvesting technologies to realize a wireless body area network (WBAN). On top of that, an intelligent agent constantly analyzes possible profiles variations, aiming at identifying physical and posture deterioration causing accidents.

Robotic manipulators have also been employed in agent-based solutions. Trainees' learning phases may be supported by formalizing and enhancing the precision and the input to be understood [23]. Relevant contributions have been provided to the interaction between therapist, trainee, and patient.

In telerehabilitation scenarios, drugs assumption correlated to a highly dynamic environment can be a recurrent situation. Mutingi et al. [10] presented an agent-based decision-making solution for drugs delivering. The bio-physiological signals the authors took into account are blood-pressure, BPM, and respiration. Elaborating the combination of such parameters and drugs therapy may provide to the medical staff important indications about patient and pathology's 
evolution. Other benefits provided by this solution are staff's workload reduction, increasing resources availability, facilitating the understanding of patients requirements and data collection.

\section{Cognitive rehabilitation}

In the scenario of cognitive rehabilitation, Abreu et al. [16] proposed a set of 3D games to rehabilitate neuropsychiatric disorders. They proposed an automatic agent-based control to facilitate the management of the software processes while the patient is playing.

Known as "the elderly silent epidemic", the Acquired Brain Injury (ABI) requires rehabilitation practices such as visuospatial, memory, functional communication, language, attention, and comprehension training [33]. Roda et al. [34] designed a MAS to (i) support the execution of the above-mentioned ABI related therapies, (ii) monitor and finally evaluate the performed activities and patient's state (e.g., stress, emotional state, BPM, and oxygen level).

Smith et al. [19] proposed another agent-based solution for functional rehabilitation involving gamification. In an environment away from rehabilitation centers, such a solution promotes a continuous, fun, and stimulating rehabilitation. Such "games" have to carefully consider a higher number of variables (e.g., incorporating expertise and motivational capacities of rehabilitation practitioners). Thus, they result in being more complex than the ones offered by off the shelf, which are typically too physically and cognitively challenging for rehabilitation patients. Information about patient compliance and progress are collected and made available to the healthcare specialists for further analysis and considerations. Moreover, the gamification technique has been exploited seeking for an enhancement of the engagement, while performing monitoring and promoting smart learning mechanisms [35].

\section{Other proposed solutions}

Providing a platform for interactive learning, Su et al. [36] developed an ontology defining vocabulary, entities and their relationships in rehabilitation medicine. Exploiting an inference engine, existing data can reveal new knowledge having an "asserted model" as input and "inferred model" as output. Another example of agent-based reasoning is presented in [37]. The authors faced two main challenges: (i) scalability - by distributing the reasoning on mobile devices, and (ii) penalization by supporting medical staff with a graphical application simplifying the definition of temporal patterns of physiological values.

Liao et al. [38] addressed reliability and security of an agent-based platform for telemonitoring.

Finally, Lai et al. [39] proposed a study involving a community scenario rather than the conventional single patient scenario. The authors evaluated the use of rehabilitation techniques for the post- or chronic-stroke survivors involving video-conferencing solutions. In conclusion, the authors praised efficacy, feasibility, and acceptability of telerehabilitation in community-dwelling stroke clients, recording improvements in both physical and psycho-social wellbeing. 


\section{Telerehabilitation: practices and requirements}

Despite the increasing awareness about the effectiveness of telerehabilitation, there is still a lack of high-quality studies evaluating types, components, modality and duration of a therapy, and the long-term functional outcomes [40].

Physiotherapists gain significant experience throughout education, training, and practice. However, the quality of a physiotherapy mainly relies on their experience. The absence of errors, information reproducibility, and simple knowledge sharing [23] still cannot be guaranteed.

According to the study conducted in collaboration with the Institute of Health in Leukerbad, a considerable amount of practices are still carried out with conventional and non-technological methods. Unfortunately, both operators and patients have experienced how easily errors or biases can be introduced in the execution (patient's side) or measurement (physiotherapist's side) of a therapy. Moreover, the use of inadequate tools or systems only complicate the rehabilitation sessions and follow up.

In order to better understand this discrepancy between current practices and physiotherapy with the support of technology, we conducted a study concluding with a questionnaire summarizing several in-person meetings.

The meetings were attended by physicians, therapists, patients and researchers. Their points of view were openly discussed focusing on various rehabilitation practices and current or desired technological supports. The questionnaire consisted of fifteen open questions (listed in the Appendix A) about the most recurring and relevant issues. The topics covered by such questions were organized in five groups:

1. joints requiring rehabilitation, related practices and adjacent limbs involved;

2. rehabilitation environment, and initial causes and conditions;

3. values and parameters that must / might / would be useful to observe;

4. common errors and limits of patients, physiotherapists, current practices and technological solutions;

5. envisioned and desired technological supports.

The outcomes of such a questionnaire are addressed, respecting the structure of the groups above.

\section{First group}

The obtained results revealed the body parts that require the most technological support that are the knee, hip, shoulder, neck and back.

\section{Second group}

To better understand pre-surgical conditions, rehabilitation environment, and what a system might be required to identify during a prevention phase, the most common causes generating the need for rehabilitation have been investigated. Lifestyle and aging have proven to be the most predominant causes. 
For example, a sedentary lifestyle might facilitate the development of arthritis/osteoarthritis and early joint degeneration, whilst an intense sportive lifestyle would cause anterior-cruciate ligament or menisci rupture and lower back pain.

\section{Third group}

Studying dynamics and physical structures, every body part that might require rehabilitation is connected or contiguous to some other regions. A crucial task is to identify which joints or limbs are eventually involved in the rehabilitation process and require observation. For example, by monitoring the movements of femur and tibia, it is possible to determine the angular interval of the knee during flexion, extension, and abduction. Summarizing the expectation expressed by the physiotherapist community, they require comprehensive solutions supporting the most significant rehabilitation practices and providing measurements if not or enhancing them to better understand:

- therapy and practice adherence;

- performance and correctness of the movements;

- possible adjustments, errors or compensations;

- coaching, encouraging and motivating the patient;

- motivation, commitment, and fatigue measurements;

- specific parameters per practice (e.g., quantification of varus or valgus thrusts during gait or jumps analysis).

\section{Fourth group}

Current available solutions in the market present critical lack of usability or information. Devices such as the kinetec [41] help the patient's knee in performing passive and continuous movements. Such a device is usually employed twice per day for a total of two hours during the acute phase ${ }^{4}$. However, the provided information on the knee angle is not precise, because the angle of the machine does not exactly correspond to the angle of the knee. Such a lack of alignment is mostly due to structural reasons, limbs misplacement or attempts to compensate the movement performed by the patient trying to reduce an undetectable pain.

Hence, the system does not offer the possibility of understanding important information such as pain, muscular resistance and patient improvement. Moreover, except for the initial assisted setup, the use of the kinetec is supposed to be unsupervised, thus enabling the propagation of all the aforementioned errors.

\section{Fifth group}

The "trust" in scientific research is a common element emerging in all the participants' answers testifying the firm believing that enhancing traditional practices with technological supports can propel patients towards a faster and better healing process. However, several functionalities are not yet available on the market.

${ }^{4}$ first phase after a surgical intervention on the knee. It is considered over when the patient is able to passively perform a $90^{\circ}$ extension 
Without any form of special commitments, the most required technological interventions are the (i) quantifying movements during rehabilitation or sports sessions, (ii) accurately measuring joint motor behavior pre-, during, and posttherapeutic intervention, (iii) qualitative assessment of the movements complementing quantitative analysis, and finally (iv) the measurement of physiological processes (e.g., cells regeneration, muscle growth and activation, blood circulation, and immunosystem condition).

Finally, to better understand the concrete possibilities of technical and technological interventions, it is worth recalling that in the context of rehabilitation, a therapy is composed of activities, an activity consists of tasks, and a task is a set of steps to be performed (e.g., gestural, postural) [34];

Considering their involvement in telerehabilitation systems, interaction tasks can be classified into four categories:

- Individual task - a task performed by a single actor (not the system).

- Collaboration task - a task carried out by two or more actors, with different or the same roles, in a collaborative way (e.g., the task could not be done without the explicit participation of each actor). Among these actors, we may find the system and/or agents, both humans and non-humans.

- Communication task - a task performed by two or more actors, with different or same roles, to exchange information. As in the previous case, among these actors, we may find the system and/or agents, both humans and non-humans.

- Coordination task - a task performed by two or more actors, with different or the same roles, that proceed in a coordinated way. As previously, among these actors, we may find the system and/or agents, both respectively human and non-human. [6]

To meet the physiotherapists' requirements and thus foster the adoption of MAS in telerehabilitation solutions, Section 4 discusses current MAS limitations and future challenges.

\section{MAS for Telerehabilitation: Discussion}

Although incorporating new technologies into rehabilitation and clinical services delivering achieved a high users' satisfaction [42], this was consistently higher for patients rather than for therapists [9].

Promised advantages brought alongside them several drawbacks. For example, deliberation time seemed to be longer, observation of physiological parameters in several solutions was neglected and was still delegated to the operator.

Fatigue, pain and overall physical state are still not easy to detect and analyze, either by a physiotherapist or a simple embedded system. Thus, a critical analysis can be: "embedded systems can read and perceive in-loco both vital and kinematic parameters mainly related with step and task execution (see Section 3), but in the case where further analysis is required, heterogeneous and proprietary (so closed) solutions have to be involved. Viceversa, expert agentbased systems can easily provide sophisticated analysis supporting therapies and activities (see Section 3), but cannot be deployed yet on embedded devices". 


\subsection{Strength}

Different patients may represent completely different scenarios. Expert agentbased systems are particularly good at modeling real-world and social systems, where problems are solved in a concurrent and cooperative way without the need of reaching optimal solutions [29]. Therefore, MAS are potentially able to dynamically relate and contextualize vital parameters and rehabilitative practices.

The adoption of MAS is crucial for activities such as decision making. Remote diagnosis, treatments adaption and planning, identification of potentially dangerous situations, and knowledge representation and manipulation are the key features common to most rehabilitative scenarios and pervasive cares [29]. Hence, such transparent and intelligent mechanisms might embed in a single comprehensive solution a broad variety of services, only limited by the "number" and "expertise/capabilities" of the involved agents.

Combining all those features, emergency mechanisms could even make decisions in the absence of a human decision maker [10]. The response time (e.g., in terms of data analysis) would be significantly reduced, especially if considering possible accuracy and consistency. However, time guarantees are still a weakness of current MAS.

The next section presents some limitations and their consequences.

\subsection{Limitations}

Current MAS cannot yet be predictable and respect strict timing constraints. The multi-agent paradigm has been embraced to solve several types of problems. However, it introduces drawbacks related to both the single application and the agent technology itself. For example, in the case of solutions involving robotic operators [23], one of the major problems is represented by a possible need to increase units.

Regarding the multi-agency, the common disadvantages already claimed in the scientific literature [10] are:

- complexity - the higher level of the systems' complexity requires more expertise and training;

- human-relationship - dealing with "virtual entities", patients are concerned about the risk of being disconnected/abandoned by the therapist;

- security - named as "possible technology perversion", this issue refer to ethical and security concerns.

Moreover, analyzing the contributions collected in Section 2.1 it is possible to notice the major problems of MAS which inhibit their adoption and limit their benefits.

Figure 1 schematically illustrates the general composition of agent-based rehabilitation systems. Indeed, MAS only appear in the "higher levels" of every system. For example, in [30] and [32] the only sections exploiting agents are the data handler, visualizer, and alert manager. In other contributions, such as [29], 


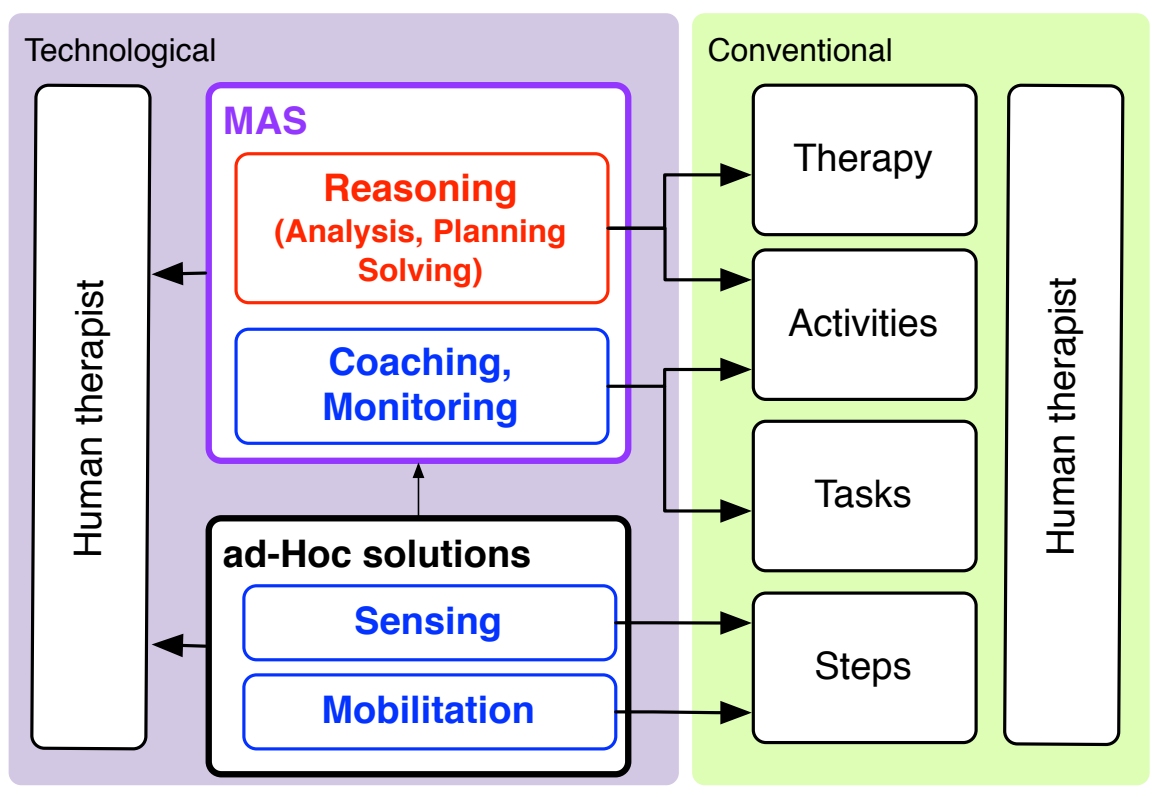

Fig. 1. Rehabilitation MAS structure.

references or details about how the agents get information from wearable sensors or embedded devices are missing or omitted.

Finally, a study involving a mobile robot hosting a multi-agent platform presented difficulties and barriers related to the use of MAS in embedded devices [43]. The authors presented a mobile robot composed of a Pandaboard, a Discovery STM32, two DC motors and a camera. The MAS running on the Pandaboard was realized using JADE [44] and was in charge of performing all the dynamics related to vision and intelligent planning. However, the robot's motion was managed by the Discovery board (unable to run the MAS and JVM due to limited resources). The "best" solution identified by the authors was to wrap the motion functionality within one of the agents, implementing a custom protocol of communication.

The limitations identified by the authors refer to:

- the need for custom communication protocols unable to respect the FIPA standard [45];

- the impossibility of running java-based agent on embedded devices;

- the difficulty of guaranteeing the respect of strict timing constraints;

- the current impossibility of running agent-based systems on real-time operating systems (e.g., Erika [46]).

Some radical changes are required to address the limitations listed above. The next section presents the critical challenges specifically required for the tel- 
erehabilitation domain.

\subsection{Future challenges}

The main challenge, involving a set of different interventions, can be stated as follows:

"bring the agents capabilities and proprieties at the sensing level."

Moving from the current rehabilitation MAS (Figure 1), which only partially exploits the agents' capabilities, towards a solution that employs agents at any level, would represent a crucial step for the multi-agent community.

For example, Figure 2 shows a possible agent-based wearable system for knee rehabilitation endorsing such a radically new challenge.

Sensing rehabilitation has to be performed at different levels, requiring diverse tasks and sensors for different situations. Delegating the sensing directly to a set of agents, spread on the wearable sensors, can produce concrete benefits. However, sensing implies understanding and correlating the exact sensors position at the exact time for the entire execution of the exercises. Employing intelligent agents in such a process mainly implies the strict consideration of constraints such as:

- timing constraints;

- scarcity of resources;

- communication means.

Timing constraints

Current MAS do not yet have mechanisms to deal with "strict timing constraints". In fact, in current implementations, their inner functionalities and interactions do not provide the possibility of facing safety-critical scenarios. As a consequence, a critical failure could lead to injuries, environmental damage, or financial losses. In the case of telerehabilitation, a delayed feedback might increase the risk of a serious injury (e.g., the patient could be requested to continue a movement over the limit of its safety range). To prevent such risks, the agents involved must be able to respect the timing constraints related to several aspects of their internal functions, as the scheduler, the negotiation and communication protocols.

\section{Scarcity of resources}

Most wearable devices have limited resources, such as memory and computational capability, to have a contained impact on the battery lifetime. Moreover, they are also subject to dimensional restrictions due to wearability and intrusivity factors. Thus, most of the conventional multi-agent frameworks cannot be deployed on such devices. Intervention to lighten agents and communication protocols are envisioned, to finally remove the barriers from the employment of 


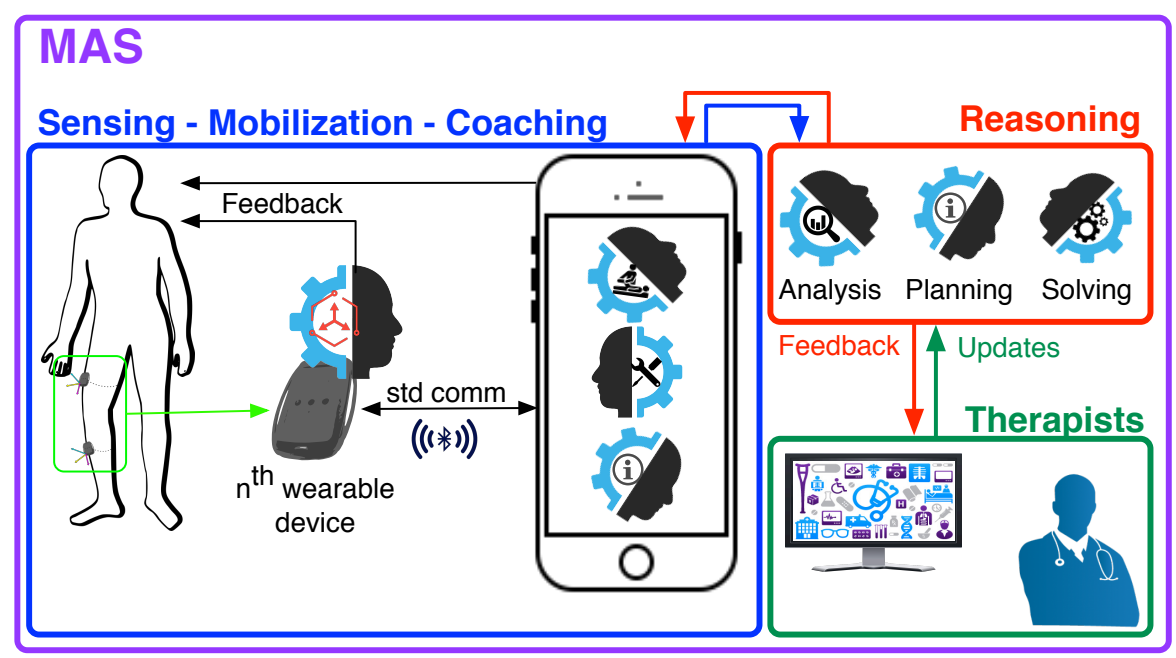

Fig. 2. Agent-based sensing: future challenge for telerehabilitation MAS.

MAS in embedded devices.

\section{Communication means}

Wearable devices for telerehabilitation are usually characterized by low-energy consumption communication means, as Bluetooth low-energy (BLE) [47] and Zigbee [48]. Standard communication protocols (e.g., FIPA ACL [45]) need to be updated to suite such new channels of communication. Indeed, broadly used in Ethernet or WiFi connection, such protocols need to take into consideration a new (and more constrictive) set of constraints. Finally, to ensure the respect of strict timing constraints, the new communication protocols must take into account boundaries and theories typical of distributed real-time applications.

Facing these new challenges require a substantial intervention within the inner mechanisms of traditional MAS. Nevertheless, the operating principles and basic protocols will still be respected, thus enabling interactions and collaborations with agents implemented according to the current policies. For example, MAS performing long-term reasoning and data handling operate in non-safety critical scenarios, which still might be implemented in the traditional way. Although incapable of guaranteeing the compliance with the newly presented constraints, such traditional agents can elaborate data provided by the real-time agents running on the wearable devices. 


\section{Conclusions}

This paper presented a comprehensive review and analysis of solutions empowering telerehabilitation. Particular emphasis has been given to agent-based systems, presenting their features, limitations, and new challenges.

Physiotherapist needs and requirements for telerehabilitation have been presented and formalized. Furthermore, the needs still left unsatisfied, with respect to conventional non-technological practices, and inadequate systems on the market, have been highlighted.

Finally, elaborating on existing rehabilitation MAS, the identified strengths are the possibility of (i) easy scenarios contextualization, (ii) facing uncertainties related to planning and problem solving, (iii) coordinating distributed sources of information, and sophisticated distributed controls. Beside these com-proved positive features, limitations such as (i) incompatibility with real-time operating systems, (ii) impossibility of running agents in embedded devices, and (iii) neglect of timing concepts within the agents' ecosystem, claimed the need for new contributions.

The most important new challenges identified in the MAS's future steps are (i) implementing time-aware mechanisms into agents' internal scheduler, communication and negotiation protocols, (ii) coping with scarcity of resources, and (iii) implementing standard protocols for new communication means.

\section{Future work}

According to Amatya et al. [40], rigorous studies are still needed for future research in appropriate outcome measures, optimal intensity, frequency, and cost effectiveness of telerehabilitation intervention over a longer period. Thus, by tightly collaborating with professional physiotherapists and researchers, we aim at facing the identified new challenges bringing the multi-agent features at the sensing level. The first expected outcomes will be primarily a fully distributed and real-time compliant MAS for knee rehabilitation, to then be employed in clinical trials and deliver appropriate studies over an extended period of time.

\section{A Questionnaire}

1) Which human joints and limbs are your (physiotherapists') primary interest?

2) What are the most typical causes/conditions? (e.g., pre-post-surgical, poststroke, or just aging-related)

Concerning the joint-limbs, you mentioned in the first question:

3) How are they treated along the four phases (acute, subacute, chronic, and maintenance)?

4) Which body parts are involved in the rehabilitation practices?

5) Which body parts must be (or should be) monitored?

Concerning the previous answers: 
6) Generally, and in your department, which are the most performed/required rehabilitative practices? (e.g., per body part - type $\& 3$ n. Of test)

7) Are they more frequently performed in ambulatory/hospital or a home/homelike environment?

8) In such practices, what is possible to observe? (e.g., extension, flexion, n. of repetitions, punctual accuracy)

9) In such practices, what is not possible to observe? (e.g., pain, fatigue, accurate evolution trend)

10) In such practices, what should and what shouldn't the patient do? (e.g., regarding position, execution-speed)

11) What are the most common errors typically performed by the patients? (e.g., compensation)

12) What are the most common errors typically performed by the physiotherapists? (e.g., misreadings)

13) What are the (human) patient limits (what should they perceive or understand, but cannot)?

14) What are the (human) physiotherapist limits (what would you like, but you cannot perceive or understand)?

15) Concerning the technological research, what do you feel is missing and needs to be implemented?

\section{Acknowledgements}

The authors wish to thank the contribution of the COST Action IC1303 Architectures, Algorithms and Platforms for Enhanced Living Environments (AAPELE).

\section{References}

1. Davide Calvaresi, Daniel Cesarini, Paolo Sernani, Mauro Marinoni, Aldo Franco Dragoni, and Arnon Sturm. Exploring the ambient assisted living domain: a systematic review. Journal of Ambient Intelligence and Humanized Computing, pages 1-19, 2016.

2. Daniel Cesarini, Davide Calvaresi, Mauro Marinoni, Paquale Buonocunto, and Giorgio Buttazzo. Simplifying tele-rehabilitation devices for their practical use in non-clinical environments. In International Conference on Bioinformatics and Biomedical Engineering, pages 479-490. Springer, 2015.

3. David Hailey, Risto Roine, Arto Ohinmaa, and Liz Dennett. Evidence of benefit from telerehabilitation in routine care: a systematic review. Journal of telemedicine and telecare, 17(6):281-287, 2011.

4. Juan Fasola and Maja Mataric. A socially assistive robot exercise coach for the elderly. Journal of Human-Robot Interaction, 2(2):3-32, 2013.

5. Štěpán Obdržálek, Gregorij Kurillo, Ferda Ofli, Ruzena Bajcsy, Edmund Seto, Holly Jimison, and Michael Pavel. Accuracy and robustness of kinect pose estimation in the context of coaching of elderly population. In 2012 Annual International Conference of the IEEE Engineering in Medicine and Biology Society, pages 11881193. IEEE, 2012. 
6. Arturo C Rodriguez, Cristina Roda, Pascual González, and Elena Navarro. Contextualizing tasks in tele-rehabilitation systems for older people. In International Workshop on Ambient Assisted Living, pages 29-41. Springer, 2015.

7. Davide Calvaresi, Daniel Cesarini, Mauro Marinoni, Paquale Buonocunto, Stefania Bandinelli, and Giorgio Buttazzo. Non-intrusive patient monitoring for supporting general practitioners in following diseases evolution. In International Conference on Bioinformatics and Biomedical Engineering, pages 491-501. Springer, 2015.

8. Dariush Mozaffarian, Emelia J Benjamin, Alan S Go, Donna K Arnett, Michael J Blaha, Mary Cushman, Sandeep R Das, Sarah de Ferranti, Jean-Pierre Després, Heather J Fullerton, et al. Executive summary: Heart disease and stroke statistics2016 update: A report from the american heart association. Circulation, 133(4):447, 2016.

9. Dahlia Kairy, Pascale Lehoux, Claude Vincent, and Martha Visintin. A systematic review of clinical outcomes, clinical process, healthcare utilization and costs associated with telerehabilitation. Disability and rehabilitation, 31(6):427-447, 2009.

10. Michael Mutingi and Charles Mbohwa. Developing multi-agent systems for mhealth drug delivery. In Mobile Health, pages 671-683. Springer, 2015.

11. Patricia A Morreale. Wireless sensor network applications in urban telehealth. In Advanced Information Networking and Applications Workshops, 2007, AINAW'0\%. 21st International Conference on, volume 2, pages 810-814. IEEE, 2007.

12. Kin Fun Li. Smart home technology for telemedicine and emergency management. Journal of Ambient Intelligence and Humanized Computing, 4(5):535-546, 2013.

13. JHM Bergmann and AH McGregor. Body-worn sensor design: what do patients and clinicians want? Annals of biomedical engineering, 39(9):2299-2312, 2011.

14. Erhan Akdoğan, Ertuğrul Taçgın, and M Arif Adli. Knee rehabilitation using an intelligent robotic system. Journal of Intelligent Manufacturing, 20(2):195-202, 2009.

15. Jiangcheng Chen, Xiaodong Zhang, and Rui Li. A novel design approach for lower limb rehabilitation training robot. In 2013 IEEE International Conference on Automation Science and Engineering (CASE), pages 554-557. IEEE, 2013.

16. Priscilla F de Abreu, Vera Maria B Werneck, Rosa Maria E Moreira da Costa, and Luis Alfredo V de Carvalho. Employing multi-agents in 3-d game for cognitive stimulation. In Virtual Reality (SVR), 2011 XIII Symposium on, pages 73-78. IEEE, 2011.

17. Sabrina Iarlori, Francesco Ferracuti, Andrea Giantomassi, and Sauro Longhi. Rgbd camera monitoring system for alzheimer's disease assessment using recurrent neural networks with parametric bias action recognition. IFAC Proceedings Volumes, 47(3):3863-3868, 2014.

18. Juan A Fraile, Javier Bajo, Juan M Corchado, and Ajith Abraham. Applying wearable solutions in dependent environments. IEEE Transactions on Information Technology in Biomedicine, 14(6):1459-1467, 2010.

19. Stuart T Smith, Amir Talaei-Khoei, Mililani Ray, and Pradeep Ray. Agent-based monitoring of functional rehabilitation using video games. In Advanced Computational Intelligence Paradigms in Healthcare 5, pages 113-141. Springer, 2010.

20. Daniel Cesarini, Pasquale Buonocunto, Mauro Marinoni, and Giorgio Buttazzo. A telerehabilitation framework for lower-limb functional recovery. In International Conference on Body Area Networks, BodyNets2014, London, UK. IEEE Computer Society, 2014.

21. Daniel Cesarini, Davide Calvaresi, Chiara Farnesi, Diego Taddei, Stefano Frediani, Bodo E Ungerechts, and Thomas Hermann. Mediation: An embedded system for 
auditory feedback of hand-water interaction while swimming. Procedia Engineering, 147:324-329, 2016.

22. Bodo Ungerechts, Daniel Cesarini, Vanessa Wiebel, and Thomas Hermann. Ears drive hands: sonification of liquid effects induced by aquatic space activities contributes to cognitive representation, 2015.

23. Sasan Adibi. Mobile health: a technology road map, volume 5. Springer, 2015.

24. Athanasios Vourvopoulos and Fotis Liarokapis. Evaluation of commercial braincomputer interfaces in real and virtual world environment: A pilot study. Computers \& Electrical Engineering, 40(2):714-729, 2014.

25. Jon Eriksson, Maja J Mataric, and C Winstein. Hands-off assistive robotics for post-stroke arm rehabilitation. In Proc. IEEE International Conference on Rehabilitation Robotics (ICORR'05), pages 21-24, 2005.

26. Ard Jacobs, Annick Timmermans, Marc Michielsen, Maaiken Vander Plaetse, and Panos Markopoulos. Contrast: gamification of arm-hand training for stroke survivors. In CHI'13 Extended Abstracts on Human Factors in Computing Systems, pages 415-420. ACM, 2013.

27. P Miranda and J Aguilar. A prototype of a multiagents system for a telemedicine environment. Engineering intelligent systems for electrical engineering and communications, 11(1):3-8, 2003.

28. Federico Bergenti and Agostino Poggi. Multi-agent systems for the application and employing of e-health services. 2010.

29. Cristina Roda, Arturo Rodríguez, Víctor López-Jaquero, Pascual González, and Elena Navarro. A multi-agent system in ambient intelligence for the physical rehabilitation of older people. In Trends in Practical Applications of Agents, MultiAgent Systems and Sustainability, pages 113-123. Springer, 2015.

30. Iker Mesa, Eider Sanchez, Javier Diaz, Carlos Toro, and Arkaitz Artetxe. Gocardio: A novel approach for mobility in cardiac monitoring. InImpact: The Journal of Innovation Impact, 6(1):110, 2016.

31. Miguel A Teruel, Elena Navarro, and Pascual González. Towards an awareness interpretation for physical and cognitive rehabilitation systems. In Ubiquitous Computing and Ambient Intelligence: 10th International Conference, UCAmI 2016, San Bartolomé de Tirajana, Gran Canaria, Spain, November 29-December 2, 2016, Proceedings, Part I 10, pages 121-132. Springer, 2016.

32. Filipe Felisberto, Nuno Costa, Florentino Fdez-Riverola, and António Pereira. Unobstructive body area networks (ban) for efficient movement monitoring. Sensors, 12(9):12473-12488, 2012.

33. Martin L Rohling, Mark E Faust, Brenda Beverly, and George Demakis. Effectiveness of cognitive rehabilitation following acquired brain injury: a meta-analytic re-examination of cicerone et al.'s $(2000,2005)$ systematic reviews. Neuropsychology, 23(1):20, 2009.

34. Cristina Roda, Arturo C Rodríguez, Víctor López-Jaquero, Elena Navarro, and Pascual González. A multi-agent system for acquired brain injury rehabilitation in ambient intelligence environments. Neurocomputing, 2016.

35. Chong Li, Zoltán Rusák, Imre Horváth, and Linhong Ji. Validation of the reasoning of an entry-level cyber-physical stroke rehabilitation system equipped with engagement enhancing capabilities. Engineering Applications of Artificial Intelligence, 56:185-199, 2016.

36. Chuan-Jun Su and Chun Wei Peng. Multi-agent ontology-based web 2.0 platform for medical rehabilitation. Expert Systems with Applications, 39(12):10311-10323, 2012 . 
37. Albert Brugués, Stefano Bromuri, Michael Barry, Óscar Jiménez Del Toro, Maciej R Mazurkiewicz, Przemyslaw Kardas, Josep Pegueroles, and Michael Schumacher. Processing diabetes mellitus composite events in magpie. Journal of medical systems, 40(2):1-15, 2016.

38. Jingsheng Liao, Chao Hu, Guan Guan, and Max Q-H Meng. An extensible telemonitoring architecture based on mobile agent method. In Robotics and Biomimetics (ROBIO), 2009 IEEE International Conference on, pages 1537-1542. IEEE, 2009.

39. Jerry CK Lai, Jean Woo, Elsie Hui, and WM Chan. Telerehabilitationa new model for community-based stroke rehabilitation. Journal of telemedicine and telecare, 10(4):199-205, 2004.

40. Bhasker Amatya, MP Galea, Jurg Kesselring, and Fary Khan. Effectiveness of telerehabilitation interventions in persons with multiple sclerosis: A systematic review. Multiple sclerosis and related disorders, 4(4):358-369, 2015.

41. Laurent Beny and René Griesmar. Device for producing continuous passive motion, December 4 2001. US Patent 6,325,770.

42. Richard M Schein, Mark R Schmeler, Andi Saptono, and David Brienza. Patient satisfaction with telerehabilitation assessments for wheeled mobility and seating. Assistive Technology®, 22(4):215-222, 2010.

43. Davide Calvaresi, Paolo Sernani, Mauro Marinoni, Andrea Claudi, Alessio Balsini, Aldo Franco Dragoni, and Giorgio Buttazzo. A framework based on real-time os and multi-agents for intelligent autonomous robot competitions.

44. Fabio Luigi Bellifemine, Giovanni Caire, and Dominic Greenwood. Developing multi-agent systems with JADE, volume 7. John Wiley \& Sons, 2007.

45. ACL Fipa. Fipa acl message structure specification. Foundation for Intelligent Physical Agents, http://www. fipa. org/specs/fipa00061/SC00061G. html (30.6. 2004), 2002.

46. Evidence. Erika enterprise rtos. http://www.evidence.eu.com.

47. Carles Gomez, Joaquim Oller, and Josep Paradells. Overview and evaluation of bluetooth low energy: An emerging low-power wireless technology. Sensors, 12(9):11734-11753, 2012.

48. ZigBee Alliance et al. Zigbee specification, 2006. 